\title{
Resíduos da laranja na elaboração de produtos alimentícios: Uma revisão
}

\author{
Orange residues in the preparation of food products: A review
}

Residuos de naranja en la preparación de productos alimenticios: Una revisión

Recebido: 23/04/2021 | Revisado: 02/05/2021 | Aceito: 04/05/2021 | Publicado: 18/05/2021

Sheyla Maria Barreto Amaral

ORCID: https://orcid.org/0000-0003-0041-5487 Instituto Federal de Educação, Ciência e Tecnologia do Ceará, Brasil E-mail: sheylaamaral82@gmail.com

Maria Jaíne Bessa

ORCID: https://orcid.org/0000-0002-6697-0355 Instituto Federal de Educação, Ciência e Tecnologia do Ceará, Brasil E-mail: jainebessa6@gmail.com

Márcia Roberta Freitas Cavalcante

ORCID: https://orcid.org/0000-0001-9934-3801 Instituto Federal de Educação, Ciência e Tecnologia do Ceará, Brasil E-mail: roberthafreitas75@gmail.com

Francisca Nayara Ferreira de Freitas

ORCID: https://orcid.org/0000-0003-0269-3835 Instituto Federal de Educação, Ciência e Tecnologia do Ceará, Brasil E-mail: nayarafreitas2010@gmail.com Marjorie Beatriz Vidal Maia

ORCID: https://orcid.org/0000-0002-3013-892X Instituto Federal de Educação, Ciência e Tecnologia do Ceará, Brasil E-mail: marjorievmaia@gmail.com

Raimundo Alves da Costa Júnior

ORCID: https://orcid.org/0000-0001-6070-0656 Instituto Federal de Educação, Ciência e Tecnologia do Ceará, Brasil E-mail: araimundoalves11@gmail.com

Marlene Nunes Damaceno

ORCID: https://orcid.org/0000-0002-3553-6740 Instituto Federal de Educação, Ciência e Tecnologia do Ceará, Brasil E-mail: marlene@ ifce.edu.br

\begin{abstract}
Resumo
O objetivo deste estudo foi compilar e analisar publicações entre os anos 2010 e 2020 que utilizaram resíduos da laranja na elaboração de produtos alimentícios. A revisão bibliográfica de literatura foi do tipo sistemática, aplicando os termos: "orange by-products", "orange peel flour", "orange albedo flour", "orange peel fiber", "orange pomace flour" e suas combinações nas línguas portuguesa e inglesa, nas bases de dados Google Acadêmico, SciELO e Periódicos CAPES. Os critérios de inclusão compreenderam o desenvolvimento de produtos alimentícios com incorporação de resíduos da laranja, a caracterização físico-química e/ou centesimal, sensorial, entre outras. Definiuse três filtros de seleção para análise: título e palavras-chave, resumo e metodologia, e leitura. Foram encontrados 468 estudos, sendo 22 o número de artigos que atenderam os critérios de seleção para a coleta e análise dos dados. Observou-se que as pesquisas são relevantes e variadas, muitos produtos já foram elaborados com resíduos da laranja: pães, bolos, biscoitos, massa, geleia, sorvetes, hambúrguer e cerveja. O aproveitamento dos resíduos da laranja originou subprodutos com melhoria nas características físico-químicas, microbiológicas, sensoriais, capacidade antioxidante e compostos bioativos. Considera-se que a utilização de resíduos da laranja é uma alternativa viável para elaboração de produtos alimentícios, com potencial de comercialização, garantindo a valorização da cultura e a redução do desperdício.
\end{abstract}

Palavras-chave: Aproveitamento; Desperdício; Farinha; Fibra; Processamento.

\section{Abstract}

The objective of this study was to collect and analyze publications between the years 2010 and 2020 that used orange residues in the preparation of food products. The literature review was systematic, applying the terms: "orange byproducts", "orange peel flour", "orange albedo flour", "orange peel fiber", "orange pomace flour" and their combinations in Portuguese and English, in the Google Scholar, SciELO and Periódicos CAPES databases. The inclusion criteria comprised the development of food products with incorporation of orange residue, physicalchemical and/or centesimal, sensory characterization, among others. Three selection filters were defined for analysis: title and keywords, abstract and methodology, and reading. It was found 468 studies, 22 the number of articles that 
met the selection criteria for data collection and analysis. It was observed that the studies are relevant and varied, many products have already been prepared with orange residue: breads, cakes, cookies, pasta, jam, ice cream, hamburger and beer. The use of orange residues resulted in by-products with improvement in physical-chemical, microbiological, sensory characteristics, antioxidant capacity and bioactive compounds. It is considered that the use of orange residues is a viable alternative for the preparation of food products, with commercial potential, ensuring the appreciation of culture and the reduction of waste.

Keywords: Use; Waste; Flour; Fiber; Processing.

\section{Resumen}

El objetivo de este estudio fue recopilar y analizar publicaciones entre los años 2010 y 2020 que utilizaron residuos de naranja en la elaboración de productos alimenticios. La revisión de la literatura fue sistemática, aplicando los términos: "orange by-products", "orange peel flour", "orange albedo flour", "orange peel fiber", "orange pomace flour" y sus combinaciones en portugués e inglés, en las bases de datos de Google Scholar, SciELO y Periódicos CAPES. Los criterios de inclusión comprendieron el desarrollo de productos alimenticios con incorporación de residuo naranja, caracterización fisicoquímica y/o centesimal, sensorial, entre otros. Se definieron tres filtros de selección para el análisis: título y palabras clave, resumen y metodología y lectura. Se encontró 468 estudios, 22 el número de artículos que cumplieron con los criterios de selección para la recolección y análisis de datos. Se observó que los estudios son relevantes y variados, ya se han elaborado muchos productos con residuos de naranja: panes, tortas, galletas, pastas, mermeladas, helados, hamburguesas y cerveza. El uso de residuos de naranja resultó en subproductos con mejora en las características fisicoquímicas, microbiológicas, sensoriales, capacidad antioxidante y compuestos bioactivos. Se considera que el uso de residuos de naranja es una alternativa viable para la elaboración de productos alimenticios, con potencial comercial, asegurando la valorización del cultivo y la reducción de desperdicios. Palabras clave: Aprovechamiento; Desechos; Harina; Fibra; Procesado.

\section{Introdução}

Estudos indicam que a laranja se originou do cruzamento do pomelo (Citrus maxima) com a tangerina (Citrus reticulata), e foi levada da Ásia para o norte da África, na Idade Média chegando ao sul da Europa, e por fim as Américas, por volta do ano 1500. A laranja é uma das frutas mais cultivadas em todo mundo, pertence à família Rutaceae, está dividida em dois grupos, a laranjeira doce e a azeda (Silva, 2015).

A produção de frutas cítricas é considerada uma das mais importantes culturas comerciais de frutas em muitos países de climas tropicais e subtropicais, como China, Brasil e Estados Unidos, sendo atualmente os primeiros países produtores de citros no mundo (Sdiri, Cuenca, Navarro, Salvador \& Bermejo, 2020). Dados da Organização das Nações Unidas para Alimentação e Agricultura (FAO, 2020) mostram que em 2019 a produção de laranja no Brasil correspondeu a 17.073.593 toneladas, ocupando a $6^{\mathrm{a}}$ posição das dez melhores commodities do país.

Segundo o relatório “O Estado da Segurança Alimentar e Nutricional no Mundo 2019”, a América Latina e o Caribe são responsáveis por $20 \%$ da quantidade total de alimentos desperdiçados no mundo desde a pós-colheita até o varejo (FAO, 2019). Sendo o principal problema no processamento de frutas e hortaliças a grande quantidade de resíduos orgânicos gerados, que ao serem desperdiçados no meio ambiente causam sérios danos, pois são de alta perecibilidade, se degradando rapidamente. Algumas empresas destinam os resíduos para elaboração de rações animais, porém, um grande número não proporciona um destino correto a esses resíduos. Diante disso, inúmeros estudos vêm sendo realizados para encontrar formas de reaproveitar corretamente os resíduos, minimizando os impactos que são gerados (Santos, Lima, Silva, Leite \& Queiroz, 2018).

No Brasil, o principal beneficiamento realizado na laranja é a elaboração do suco concentrado para exportação e consumo interno, os resíduos gerados nesse processamento correspondem aproximadamente a metade de cada fruto utilizado, representados pelas cascas, sementes e resíduos de polpa. Porém, não é do conhecimento de todos que esses resíduos apresentam óleos essenciais, carboidratos solúveis, celulose, propectina, pectina, flavonoides, aminoácidos, essências aromáticas e vitamina $\mathrm{C}$, podendo ser utilizados para obtenção de uma gama de produtos como: doces, geleias, bebidas, farinhas, pães, biscoitos, bolos, entre outros (Benelli, 2010). 
O aproveitamento de resíduos alimentícios para elaboração de derivados, além de fornecer produtos a baixo custo, também pode apresentar qualidade nutricional e sensorial semelhantes ao produto de origem ou a matéria-prima, ou ainda atender a demanda de mercado de alimentos de fácil e rápido preparo e consumo (Araújo \& Budoia, 2019).

Diante do exposto, o objetivo do presente estudo foi compilar e analisar os artigos publicados nos anos 2010 a 2020 que realizaram o aproveitamento de resíduos da laranja, desenvolvendo e caracterizando subprodutos.

\section{Metodologia}

\subsection{Caracterização do estudo}

O presente trabalho se configura em uma revisão bibliográfica de literatura, do tipo sistemática, com propósito de agrupar estudos que utilizaram as partes residuais do fruto da laranjeira na incorporação em produtos alimentícios.

Segundo Biolchini, Mian, Natali, Conte e Travassos (2007) a revisão bibliográfica sistemática (RBS) serve de instrumento para estruturar trabalhos publicados em um determinado tema, proporcionando aos autores a elaboração de um resumo do conhecimento existente sobre o assunto pesquisado. O modelo para condução de uma RBS proposto pelos autores abrange as etapas: planejamento, execução e análise dos resultados.

\subsection{Estratégia de busca}

A coleta dos dados foi realizada no período de janeiro a março de 2021, e as bases de dados utilizadas para a compilação dos artigos científicos foram: Google Acadêmico, SciELO e Periódicos Capes. Buscou-se por artigos publicados e indexados nos referidos bancos de dados, entre os anos 2010 e 2020.

As pesquisas foram realizadas utilizando os seguintes termos: "subprodutos da laranja", "farinha da casca da laranja", "farinha do albedo da laranja", "fibra da casca da laranja", e "farinha do bagaço da laranja", orange by-products", "orange peel flour", "orange albedo flour”, "orange peel fiber”, “orange pomace flour” e suas combinações nas línguas portuguesa e inglesa.

\subsection{Critérios de seleção}

Para o primeiro critério de inclusão foram pesquisados estudos que abordassem o desenvolvimento de produtos alimentícios com incorporação de resíduos da laranja em sua composição e realizassem a caracterização físico-química e/ou centesimal, análise sensorial, entre outras determinações no produto final desenvolvido.

Como segundo critério, aplicou-se filtros para encontrar estudos que atendessem ao objetivo do trabalho em questão. O primeiro filtro de seleção avaliou o título e as palavras-chave, e o segundo filtro aplicado foi a leitura do resumo e da metodologia. No terceiro e último filtro, fez-se a leitura completa dos artigos selecionados.

Já os critérios de exclusão envolveram artigos de pesquisa publicados em anais de eventos, monografias, dissertações, teses e editoriais de jornais, artigos de revisão, capítulos de livros; trabalhos sem relação direta com a temática da pesquisa; e os estudos duplicados nas bases de dados.

\subsection{Processo de seleção dos artigos e coleta dos dados}

As buscas nas bases de dados geraram um total de 468 artigos que foram avaliados pelo título e palavras-chave resultando em 51 estudos, nos quais foram examinados o resumo e a metodologia, restando 30 trabalhos que após a exclusão das não conformidades em relação a temática definida, e dos artigos repetidos nas bases de dados, resultaram na amostra do estudo. Após avaliação minuciosa e objetiva, selecionou-se 22 artigos que preenchiam os critérios apresentados sendo estes avaliados de forma integral (Figura 1). 
As principais informações nos estudos selecionados para esta revisão estão detalhadas no Quadro 1. Com o intuito de facilitar a discussão dos resultados, os artigos foram identificados através dos códigos A1 a A22.

Figura 1 - Processo de seleção dos artigos na revisão bibliográfica sistemática.

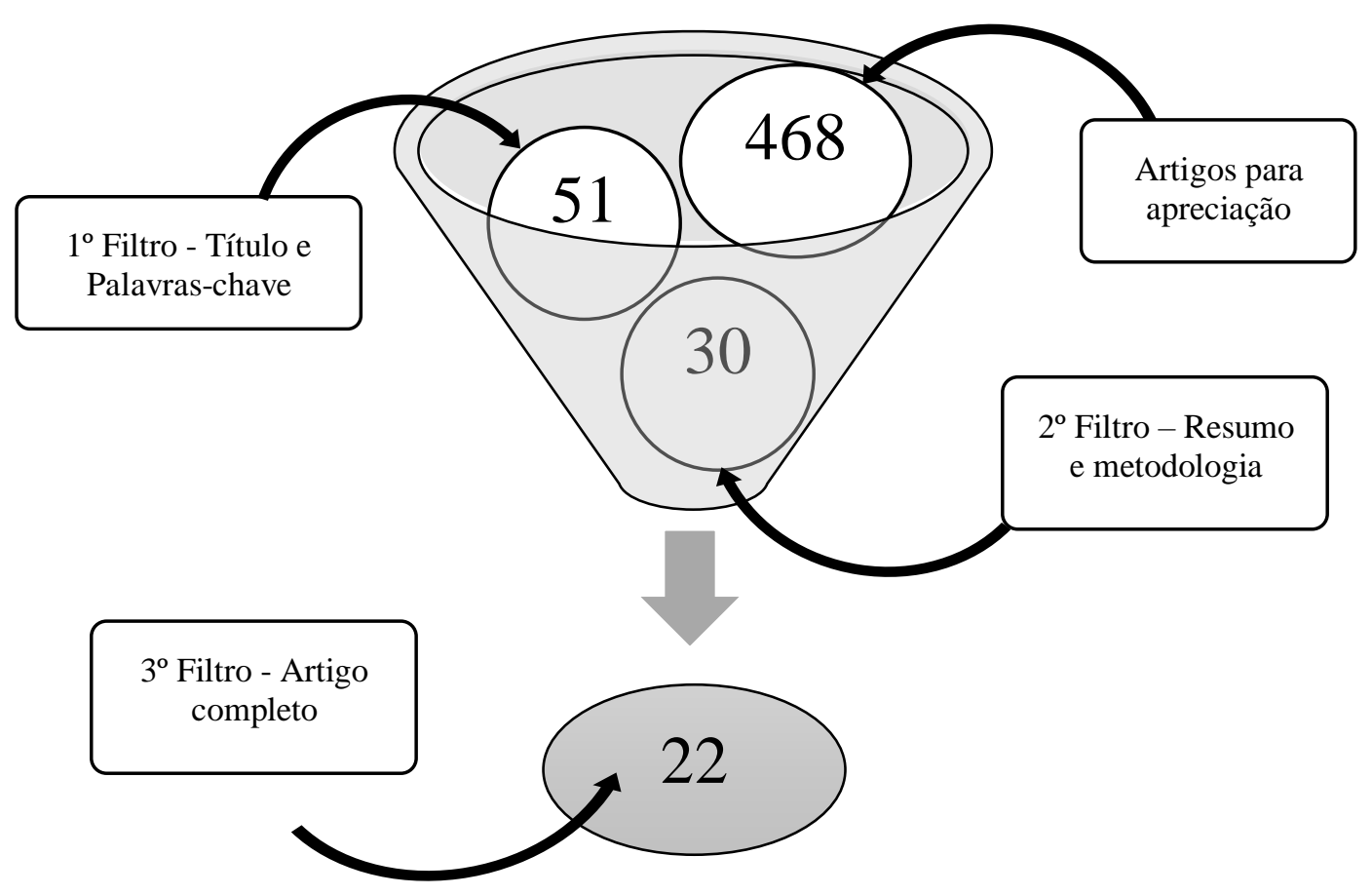

Fonte: Autores (2021). 
Quadro 1 - Características dos artigos selecionados.

\begin{tabular}{|c|c|c|c|c|c|}
\hline \multirow[b]{2}{*}{ ID } & \multicolumn{5}{|c|}{ Coleta dos Dados } \\
\hline & Resíduo incorporado & $\begin{array}{l}\text { Subproduto } \\
\text { elaborado }\end{array}$ & Análise realizada & Base de dados & Referência \\
\hline A1 & Farinha do albedo & Pão de forma e biscoito & Sensorial & Google Acadêmico & Silva et al. (2016) \\
\hline A2 & Farinha do bagaço & Pão & Microbiológica, centesimal e sensorial & Google Acadêmico & Storrer et al. (2017) \\
\hline $\mathbf{A 3}$ & Farinha da casca & Pão de mel & Microbiológica e sensorial & Google Acadêmico & Nogueira et al. (2018) \\
\hline A4 & Farinha do bagaço & Muffins & Centesimal, física e sensorial & Google Acadêmico & Romero-Lopez et al. (2011) \\
\hline A5 & Farinha do albedo & Bolo & Centesimal e sensorial & Google Acadêmico & Silva et al. (2012) \\
\hline A6 & Farinha do albedo & Bolo & Centesimal e sensorial & SciELO & Silva et al. (2013) \\
\hline A7 & Farinha do albedo & Bolo & Centesimal, física e sensorial & Periódicos Capes & Oliveira et al. (2016) \\
\hline A8 & Farinha da casca e do bagaço & Bolo & Sensorial e cálculo nutricional & Google Acadêmico & Urrutia et al. (2019) \\
\hline A9 & Farinha do albedo & Biscoito & Centesimal e sensorial & Periódicos Capes & Santos et al. (2010) \\
\hline $\mathbf{A 1 0}$ & Farinha do albedo & Biscoito & Centesimal e sensorial & Periódicos Capes & Santos et al. (2011) \\
\hline A11 & Farinha da casca e bagaço & Biscoito & Centesimal e sensorial & Google Acadêmico & Tozatti et al. (2013) \\
\hline A12 & Farinha da casca & Biscoito & Físico-química e sensorial & Google Acadêmico & Rosolen et al. (2018) \\
\hline A13 & Farinha da casca & Biscoito & Centesimal, física, funcional, fitoquímica e sensorial & Google Acadêmico & Ayo, Ayo e Igweaka (2018) \\
\hline A14 & Farinha da casca & Biscoito & Físico-química e sensorial & Periódicos Capes & Ramos et al. (2020) \\
\hline A15 & Farinha da casca & Marmelada & Físico-química e atividade antioxidante & Periódicos Capes & Sicari et al. (2018) \\
\hline A16 & Farinha do albedo & Hambúrguer & Centesimal, físico-química e sensorial & Periódicos Capes & Silva et al. (2020) \\
\hline A17 & Farinha do albedo, do flavedo, e & Sorvete & Centesimal e sensorial & SciELO & Oliveira, Winkelmann e Tobal (2019) \\
\hline
\end{tabular}




\begin{tabular}{|c|c|c|c|c|c|}
\hline \multirow[b]{2}{*}{ ID } & \multicolumn{5}{|c|}{ Coleta dos Dados } \\
\hline & Resíduo incorporado & $\begin{array}{l}\text { Subproduto } \\
\text { elaborado }\end{array}$ & Análise realizada & Base de dados & Referência \\
\hline & das sementes & & & & \\
\hline A18 & Fibra da casca & Sorvete & Física, química e sensorial & SciELO & Boff et al. (2013) \\
\hline A19 & $\begin{array}{l}\text { Fibra da casca, do bagaço e das } \\
\text { sementes }\end{array}$ & Sorvete & Física, química e sensorial & SciELO & Crizel et al. (2014) \\
\hline A20 & $\begin{array}{l}\text { Fibra da casca, do bagaço e das } \\
\text { sementes }\end{array}$ & Massa fettuccini & Física, química e sensorial & SciELO & Crizel et al. (2015) \\
\hline A21 & Fibra da casca & Pão de forma & Física, físico-química e sensorial & SciELO & Stoll, Flôres e Thys (2015) \\
\hline A22 & Casca & Cerveja & $\begin{array}{l}\text { Físico-química, fenólicos totais, atividade } \\
\text { antioxidante, microbiológica e sensorial }\end{array}$ & SciELO & Pereira et al. (2020) \\
\hline
\end{tabular}

Fonte: Autores (2021). 


\section{Resultados e Discussão}

\subsection{Processamento de pães e massas}

O estudo de Silva et al. (2016), identificado como A1, utilizou a laranja lima e seus resíduos para o desenvolvimento de novos produtos. Quanto ao aproveitamento das partes residuais, a farinha do albedo foi utilizada para substituir a farinha de trigo em dois produtos: pão de forma (2,5\% e 5,0\%) e biscoitos macerados e não macerados (5\%, 15\% e 20\%). Em seguida, realizaram análise sensorial, para avaliar a aceitação nos atributos aparência, textura, cor, sabor e aroma. Encontraram que a maceração possibilitou a retirada do amargor nos biscoitos, conferindo-lhes maior aceitação por parte dos avaliadores. Já os biscoitos sem maceração obtiveram Índice de Aceitabilidade inferior a 70\%, resultado associado ao sabor amargo existente. Os pães de forma não apresentaram diferença significativa entre si, quanto aos atributos avaliados, contudo, foi perceptível a melhora na apreciação do aroma e da cor à medida que se aumentava o teor de farinha de albedo na formulação. Os autores concluíram que a utilização do albedo na elaboração de farinha e sua incorporação em produtos de panificação é uma alternativa viável de comercialização e aproveitamento deste resíduo; recomendaram a utilização do chocolate, para mascarar o amargor em caso de formulações elaboradas com maiores concentrações da farinha do albedo.

A pesquisa de Storrer, Meireles, Perly, Lima e Mattanna (2017), identificada como A2, aproveitou o bagaço da laranja em forma de farinha e incorporou (7\%) em uma receita tradicional de pão. Após o processamento, realizaram análises microbiológicas e de composição centesimal na farinha e no pão e teste sensorial no produto final. Com relação à avaliação microbiológica, tanto a farinha como o pão apresentaram qualidade higiênico satisfatória, conforme os parâmetros recomendados pela legislação. O pão apresentou um elevado teor lipídico $(7,94 \%)$ considerando esse tipo de produto e em comparação à farinha que foi bastante inferior $(0,34 \%)$. O teor proteico foi de $9,01 \%$, acima do preconizado pela Tabela Brasileira de Composição de Alimentos $(8,4 \%)$ para pão, resultado bastante satisfatório. A avaliação sensorial do pão demonstrou todas as médias dentro da região de aceitação do teste: 6,71; 7,10; 7,65; 7,59 e 7,46 para aroma, sabor, textura, aparência geral e cor, respectivamente, e Índice de Aceitabilidade acima de 70\% para todos os atributos. Os autores concluíram que o pão produzido com farinha do bagaço da laranja possui viabilidade de comercialização e consumo, diante do baixo custo de aquisição do resíduo, da ótima aceitabilidade sensorial e do seu alto teor de proteínas. Ressaltaram também os benefícios proporcionados à saúde e ao meio ambiente quando se faz o aproveitamento adequado desses resíduos.

O trabalho de Nogueira et al. (2018), identificado como A3, incorporou farinha da casca de laranja (FCL) em pão de mel. Foram elaboradas três formulações, a padrão, sem a adição da farinha, e duas formulações com incorporação de $25 \%$ e 50\%. Em seguida, avaliaram-se as características microbiológicas e sensoriais do produto. Os resultados dos testes microbiológicos indicaram que os pães elaborados possuíam qualidade higiênico satisfatória, dentro dos padrões permitidos pela legislação. Quanto à análise sensorial, o único atributo que apresentou diferença significativa entre os tratamentos foi a cor. Para preferência, a formulação com 50\% FCL foi a mais preferida pela maioria dos avaliadores, seguida da formulação com 25\% e em terceiro a formulação padrão. Para o teste CATA (Check-All-That-Apply), os pães com 50\% FCL apresentaram o descritor "Sabor Marcante", e os pães com 25\% FCL os descritores "Firme" e "Úmido" dentre os 20 descritores elencados na ficha. Concluiu-se que todas as formulações obtiveram boa aceitação, mas os pães elaborados com maior incorporação da farinha da casca de laranja se destacaram em relação ao sabor marcante e a harmonia com as especiarias.

O estudo de Crizel, Rios, Thys e Flôres (2015), identificação A20, avaliou o impacto da incorporação da fibra da casca, bagaço e sementes de laranja em massas frescas tipo fettuccine. Foram elaboradas quatro formulações: FP0, FP25, FP50 e FP75 com 0\%, 2,5\%, 5,0\% e 7,5\% de fibra, respectivamente. Em seguida foram avaliadas as propriedades nutricionais, culinárias e sensoriais das massas. Não houve diferença significativa entre as formulações para o conteúdo de umidade, proteínas, lipídeos e carboidratos. O teor de fibra alimentar total da FP75 foi 7,50\%, aumento correspondente a 99\% em 
comparação a formulação controle (3,77\%), sendo considerado um produto com "alto teor de fibras". O teor de fenólicos totais de FP75 (1,68 mg/g) também apresentou aumento significativo em comparação a FP0 (1,19 mg/g). A incorporação da fibra não alterou as propriedades de qualidade do cozimento, nem afetou os parâmetros de cor das massas. Quanto ao teste sensorial, à medida que aumentava a porcentagem de fibra na formulação, houve diminuição das médias nos atributos de aceitação geral, sabor e gosto residual, associado ao amargor proveniente da fibra. Diante disso, os autores consideraram a incorporação da fibra da laranja em massas frescas uma alternativa viável, tendo em vista o aumento da capacidade antioxidante e do teor de fibras, não alterando as características de qualidade do produto. Contudo, novos estudos são necessários, realizando um prétratamento nas fibras com intenção de amenizar o sabor amargo característico desse subproduto.

A pesquisa de Stoll, Flôres e Thys (2015), identificação A21, elaborou pão de forma utilizando a fibra da casca da laranja amarga como substituto da gordura. Seis formulações foram desenvolvidas variando as concentrações de água, fibra da casca, enzima $\alpha$-amilase e gordura vegetal hidrogenada. Na fibra foi avaliada a capacidade de retenção de água; e nos pães: volume, atividade de água, coloração do miolo, análise sensorial, e acompanhamento da vida de prateleira, através do índice de retrogradação do amido por sete dias. A formulação F5 (2,5\% da fibra e 30 ppm da enzima) produziu pães com fibras, livres de gordura, volume desejado, sabor e textura aceitáveis, e aproximadamente $80 \%$ de aceitação global. A coloração do miolo dos pães com adição da fibra apresentou aumento significativo na coordenada $b^{*}$, acentuando-se sua cor amarela. A associação da fibra com a $\alpha$-amilase conservou características de vida útil dos pães, mantendo a atividade de água nos sete dias de armazenamento. Concluiu-se que a fibra da laranja pode substituir a gordura em produtos de panificação, sem alteração da qualidade e composição, como nos pães de forma elaborados, demonstrando potencial de comercialização.

\subsection{Produção de bolos}

Romero-Lopez, Osorio-Diaz, Bello-Perez, Tovar e Bernardino-Nicanor (2011), identificação A4, elaboraram farinha do bagaço de laranja doce e incorporaram em muffins (bolinho inglês), variando as concentrações em 0\% (controle), 10\% e 15\%. Em seguida, foi realizada a determinação da composição centesimal; fração de amido total, de digestão rápida, de digestão lenta e resistente; cinética de digestão de amido in vitro e análise sensorial. A farinha do bagaço incorporada nos muffins aumentou o teor de fibra dietética total (FDT) em 40\% e 63\%, em comparação com a formulação controle. Os teores de amido de digestão lenta também aumentaram, já os de digestão rápida, os níveis de amido resistente e o índice glicêmico diminuíram nas amostras com a farinha. O produto com adição de $10 \%$ da farinha apresentou aceitação sensorial semelhante ao controle, porém, para a amostra com $15 \%$ a pontuação de aceitabilidade foi menor. Os altos níveis de FDT e da fração indigestível obtidos indicaram que os muffins são uma alternativa potencial para incorporação na dieta de pessoas que possuem diferentes necessidades nutricionais.

O estudo de Silva, Santos, Alves, Batista e Marcellini (2012), identificação A5, realizou a otimização de bolo de chocolate substituindo parcialmente a farinha de trigo por fécula de mandioca e farinha do albedo da laranja. Realizou-se teste sensorial variando as concentrações de fécula, farinha e açúcar, através de planejamento fatorial, para a escolha da formulação otimizada. Após avaliação das médias dos atributos obtidas nos 18 ensaios, selecionaram a formulação com 27,5\% de fécula, 7,5\% de farinha do albedo e $110 \%$ de açúcar. Por último, avaliou-se a aceitação sensorial e composição centesimal das formulações padrão (100\% trigo), comercial e otimizada. Para a composição centesimal, merece destaque o teor de fibra bruta da formulação otimizada $(2,81 \%)$ em relação a padrão $(1,03 \%)$. Quanto à aceitação, os atributos impressão global, sabor e textura, não apresentaram diferença significativa entre as formulações. Os índices de aprovação foram superiores a $48 \%$ e índices de rejeição inferiores a $21 \%$. Concluiu-se que a farinha do albedo ao substituir de forma parcial a farinha de trigo não causou mudanças significativas na composição nutricional e na aceitação sensorial do bolo de chocolate elaborado, expressando-se o potencial de comercialização do produto sem comprometer suas características tecnológicas e de qualidade. 
No trabalho de Silva et al. (2013), identificação A6, produziu-se farinha do albedo de laranja doce, utilizando na elaboração de bolo de chocolate, com intuito de oferecer um produto economicamente viável e com melhor qualidade nutricional. Realizou-se teste sensorial para avaliar a influência da concentração de açúcar, polvilho azedo e farinha do albedo, através de planejamento fatorial com 18 ensaios. A formulação otimizada escolhida foi de 27,5\% de polvilho, $6 \%$ de farinha de albedo e $130 \%$ de açúcar. Avaliou-se a composição centesimal e sensorial nas formulações comercial, padrão (100\% trigo) e otimizada. Os resultados da avaliação centesimal apontaram o aumento de $73,79 \%$ de fibra bruta na formulação otimizada em relação à padrão. As formulações não diferiram significativamente entre si nos atributos sensoriais e apresentaram índices de aprovação superiores a $50 \%$ e índices de rejeç̧ão inferiores a $20 \%$. Os autores concluíram então que o bolo elaborado apresentou boas características sensoriais e a utilização da farinha de albedo somada ao polvilho em substituição parcial à farinha de trigo é uma alternativa favorável na elaboração de produtos panificáveis.

O estudo de Oliveira et al. (2016), identificação A7, realizou avaliação física, centesimal e sensorial de bolos com resíduos de maracujá e laranja. Utilizaram a farinha do albedo de ambas as frutas, como forma de aproveitamento dos resíduos, elaborando quatro formulações: farinha de trigo (FT); FT e $20 \%$ de farinha de maracujá (FM); FT e 12,5\% de farinha de laranja (FL); FT, 10\% de FM e 6,25\% de FL. O bolo com FT, FM e FL apresentou maior umidade (31,49\%). A formulação com $20 \%$ de farinha de maracujá apontou maior teor de fibra alimentar (4,95\%), seguida da amostra com as três farinhas (3,92\%). Em relação aos aspectos físicos, o bolo com farinha de laranja e farinha de maracujá apresentou características semelhantes ao bolo padrão, resultado desejável, já que a substituição do glúten não afetou sua qualidade tecnológica. Quanto ao teste sensorial, o bolo com farinha do albedo de laranja se destacou, pois não diferiu significativamente da amostra padrão em todos os atributos avaliados, e apresentou as maiores médias para cor e aroma. Concluiu-se que é possível adicionar farinhas de resíduos de frutas, e obter produtos enriquecidos nutricionalmente, por apresentarem altos teores de fibra alimentar. Contudo, os atributos de cor e sabor necessitam ser melhorados, em busca de amenizar o sabor amargo característico das frutas cítricas.

A pesquisa de Urrutia et al. (2019), identificada como A8, teve por objetivo elaborar bolos com incorporação de farinhas de subprodutos da laranja pera e farinha de aveia em substituição à farinha de trigo. Foram elaboradas três formulações: F1 com 27\% de farinha de trigo, F2 com 0,4\% de farinha da casca da laranja e 16,4\% de farinha de aveia, e F3 com $0,4 \%$ de farinha da casca e bagaço da laranja e 16,4\% de farinha de aveia. Seguindo-se a avaliação sensorial e o cálculo nutricional dos bolos. A formulação F1 teve melhor aceitação que F2 e F3, diferindo significativamente entre os atributos impressão global, aparência, aroma, textura e sabor, possivelmente relacionado a utilização da farinha de trigo na formulação. Entretanto, os bolos com adição de farinha de aveia e de resíduos da laranja se destacaram do ponto de vista nutricional, com menor teor de carboidratos simples (32,2\%). A formulação F3 teve menor aceitação em relação ao sabor, pois a presença do bagaço da fruta conferiu-lhe um sabor mais amargo. Concluiu-se que a elaboração de bolos com incorporação de resíduos da laranja é uma forma de reduzir o desperdício de alimentos e oferecer produtos ricos em nutrientes com características funcionais, proporcionando benefícios à saúde dos consumidores e ao meio ambiente.

\subsection{Preparo de biscoitos}

Santos et al. (2010), identificação A9, desenvolveram em seu estudo biscoitos de chocolate com misturas de farinha de trigo, fécula de mandioca e farinha do albedo da laranja, para agregar valor econômico ao resíduo e nutricional ao produto. A escolha da formulação otimizada foi realizada por avaliação sensorial no planejamento fatorial $2^{3}$, no qual as variáveis independentes foram a concentração de fécula, açúcar e farinha do albedo em 18 ensaios. Após análise dos dados e seleção da formulação otimizada, avaliou-se a composição centesimal e sensorial das amostras: comercial, padrão e otimizada (27,5\% de fécula, $100 \%$ de açúcar e 7,5\% de farinha de albedo). A farinha do albedo conferiu ao biscoito da formulação otimizada $2,11 \%$ 
de fibra bruta, teor quase quatro vezes superior ao presente no padrão $(0,56 \%)$. Os biscoitos com farinhas mistas apresentaram bons níveis de aceitação para os atributos avaliados (impressão global, aparência, aroma, textura e sabor), com escores entre 5 e 8 na escala hedônica e apresentaram índices de aprovação superiores a 44\% e índices de rejeição inferiores a 30\%. Concluiuse que a utilização de farinha de albedo de laranja na elaboração de biscoitos é favorável, pois além dos testes sensoriais indicarem uma boa aceitação, agregou ao produto um teor de fibra considerável, proporcionando enriquecimento nutricional.

Santos et al. (2011), identificação A10, utilizaram o polvilho azedo e farinha de albedo da laranja como substitutos da farinha de trigo na elaboração de biscoitos de chocolate. Dezoito ensaios avaliaram a influência da concentração de açúcar, polvilho azedo e farinha do albedo na aceitação sensorial para escolha da formulação otimizada por planejamento experimental $2^{3}$. Após análise dos resultados e seleção da formulação otimizada, realizou-se a avaliação da composição centesimal e sensorial das amostras: comercial, padrão e otimizada (35\% de polvilho, $100 \%$ de açúcar e 7,5\% de farinha de albedo). Os biscoitos apresentaram escores sensoriais entre 5,6 e 7,2 para todos os atributos avaliados (impressão global, aparência, aroma, textura e sabor). Com relação ao teste de intenção de compra, as formulações padrão e otimizada diferiram da formulação comercial. O biscoito com farinha do albedo obteve 3,08\% de fibra bruta, podendo ser classificado como alimento "fonte de fibras", já a formulação padrão apresentou apenas $0,56 \%$. Concluiu-se que a adição de polvilho azedo e albedo da laranja permitiu a elaboração de um alimento com elevado teor de fibras, sem comprometimento dos atributos sensoriais.

Tozatti, Rigo, Bezerra, Córdova e Teixeira (2013), identificação A11, elaboraram farinha a partir dos resíduos (casca e bagaço) da laranja pera e utilizaram em substituição parcial da farinha base (70\% de farinha de trigo; $15 \%$ de flocos de batata; $10 \%$ de amido de milho; $5 \%$ de fécula de mandioca) no desenvolvimento de biscoitos tipo cracker. Quatro formulações foram preparadas: $0 \%$ (padrão), $5 \%, 10 \%$ e $20 \%$ da farinha de resíduos. Realizou-se a caracterização sensorial e centesimal. O teste de aceitação indicou a formulação com $5 \%$ da farinha de resíduos com maior média $(7,25)$ entre as formulações com a farinha de resíduos. A formulação com 5\% de farinha apresentou 7,54\% de fibra bruta, acréscimo de $101 \%$ em relação a padrão $(3,75 \%)$, sendo o biscoito considerado um alimento com "alto teor de fibras". Concluiu-se que o biscoito elaborado com farinha da casca e bagaço da laranja é uma boa alternativa de consumo saudável e funcional, possibilitando o aproveitamento desses resíduos pela indústria alimentícia na elaboração de produtos com qualidade nutricional e sensorial semelhantes aos encontrados no mercado.

Rosolen et al. (2018), identificação A12, produziram e avaliaram biscoitos tipo cookies com substituição parcial da farinha de trigo por farinha da casca da laranja, para o emprego do resíduo de forma saudável. Foram elaboradas quatro formulações: controle (100\% de farinha de trigo) e 10\%, $20 \%$ e $30 \%$ da farinha da casca da laranja. Em seguida, avaliaram sua composição físico-química e sensorial. Observou-se que o aumento da concentração da farinha da casca da laranja, promoveu aumento no teor de minerais, sendo os mais abundantes: potássio, sódio e cálcio, indicando a influência da utilização de diferentes farinhas na composição nutricional de um alimento. A análise sensorial indicou diferença significativa entre as formulações para impressão global, sabor e odor, inferindo que o aumento da concentração da farinha da casca exerceu influência nesses atributos, mas não interferiu na aparência e textura dos cookies. Quanto ao Índice de Aceitabilidade, obtevese: $85,3 \%, 89,9 \%$ e $70,1 \%$ para as formulações com $10 \%, 20 \%$ e $30 \%$ da farinha da casca da laranja, respectivamente. Concluiu-se que a utilização da casca da laranja para elaboração de farinha e incorporação em produtos de panificação possibilita a redução dos desperdícios e danos causados ao meio ambiente, além de produzir alimentos com elevado teor nutricional e alta aceitabilidade.

O estudo de Ayo, Ayo e Igweaka (2018) identificação A13, teve por objetivo elaborar biscoito à base de farinha de grãos de acha (cereal africano) com substituição da farinha da casca de laranja, visando sua melhoria nutricional. Foram elaboradas seis formulações substituindo a farinha de acha pela farinha de laranja em 0\%, 5\%, 10\%, 15\%, 20\% e 25\%. Em seguida, avaliaram a composição centesimal, física, funcional, fitoquímica e sensorial dos produtos. $\mathrm{O}$ aumento da farinha da 
casca de laranja elevou os teores de umidade, fibras, cinzas, proteínas e lipídios, bem como, das propriedades físicas e funcionais dos biscoitos. As concentrações de carotenoides, saponinas, flavonoides e antocianinas aumentaram com a adição da farinha da casca de laranja nas formulações, chegando a 9,44 mg/100 g de carotenoides. Esses resultados conferem aos biscoitos propriedades antioxidantes, anticancerígenas, anti-inflamatórias e antimutagênicas. O biscoito com substituição de $5 \%$ foi mais aceito com pontuações médias de cor, textura, sabor, flavor e aceitabilidade geral de 7,55, 7,65, 7,30, 7,30 e 7,05, respectivamente. Concluiu-se que os biscoitos são alternativas viáveis de consumo e comercialização, pois apresentaram boa aceitação e consideráveis teores de compostos fitoquímicos que conferem benefícios à saúde humana.

O trabalho de Ramos, Pereira, Andressa, Schmiele e Amaral (2020), identificado como A14, consistiu na produção de farinhas a partir de resíduos de frutas (casca de abacaxi, de manga, de banana, de laranja, entrecasca de melancia e amêndoa da manga) para adicionar em biscoito tipo cookie. Foram elaboradas formulações padrão e com substituição de $15 \%$ da farinha de trigo por cada tipo de farinha de resíduo. Avaliou-se nas farinhas o rendimento, pH e cor, e nos cookies: volume específico, cor, textura e aceitação sensorial. A farinha da casca da laranja apresentou pH de 4,68, abaixo da neutralidade, característica ácida proveniente da fruta. Ao substituir a farinha de trigo pela farinha da casca da laranja obteve-se $20,8 \%$ de rendimento. Com relação aos parâmetros de cor dos cookies, identificou-se diferenças entre a coloração da formulação padrão e da formulação com a farinha, influenciada pelo resíduo incorporado. Os cookies produzidos com a farinha da casca da laranja apresentaram escores inferiores à formulação padrão, com gosto levemente amargo, influenciando na aceitação do produto final. Concluiu-se que é possível elaborar um produto nutritivo e saboroso utilizando resíduos de frutas, promovendo uma alimentação mais saudável aos consumidores e reduzindo o desperdício dos resíduos alimentícios.

\subsection{Desenvolvimento de sorvete}

No trabalho de Oliveira, Winkelmann e Tobal (2019), identificação A17, produziram-se farinhas dos resíduos de laranjas sanguíneas-de-mombuca, utilizando o albedo (FA), o flavedo (FF) e as sementes (FS). Cinco formulações de sorvete de chocolate foram elaboradas substituindo a gordura pelas farinhas: padrão (5,8\% de creme de nata), F1 (1,4\% da mistura de FF e FS), F2 (1,9\% da mistura de FF e FS), F3 (2,0\% de FA) e F4 (3,0\% de FA). Após o processamento, realizou-se a composição centesimal e a aceitação sensorial. Inicialmente, avaliou-se a composição centesimal das farinhas, com destaque para o teor de fibra, de 21\%, 26,9\% e 35\%, para FF, FA e FS, respectivamente. Em seguida, os sorvetes foram submetidos ao teste sensorial, observando que a formulação F4 obteve as maiores médias de aceitação entre as formulações com farinha. Seguiu-se com a análise centesimal das formulações padrão e F4. Não houve diferença significativa para umidade, carboidratos, proteínas e cinzas. Destacando o aumento no teor de fibras e a diminuição da gordura na amostra F4 em relação ao sorvete padrão, considerando-se a formulação com farinha do albedo mais aceitável. Concluiu-se que as farinhas são uma alternativa viável na elaboração de novos alimentos, diante da sua composição nutricional, bem como, a possibilidade da extração de fibras através dos resíduos da laranja, que na maioria dos casos são desperdiçados, causando danos ao meio ambiente e à saúde da população.

Boff, Crizel, Araujo, Rios e Flôres (2013), artigo identificado por A18, utilizaram a fibra da casca da laranja amarga como substituto de gordura na elaboração de sorvete de chocolate. Três formulações foram elaboradas, a controle, F1 e F2 com $0 \%, 0,74 \%$ e $1,10 \%$ de fibra, respectivamente. Em seguida foi realizada a determinação da composição centesimal, valor energético, overrun, taxa de derretimento e análise sensorial. A umidade variou entre $63 \%$ e $70 \%$, e a amostra controle apresentou menor umidade e maior teor lipídico em comparação com F1 e F2. A adição de fibra auxiliou na diminuição da velocidade de derretimento do sorvete e reduziu o percentual de overrun, proporcionando diminuição da incorporação de ar. O índice de aceitabilidade das amostras com adição de fibra foi de $78 \%$. A adição da fibra não afetou as características físicas e químicas do sorvete e reduziu o valor energético total, sendo classificado como light, já que a presença de fibra reduziu em 
mais de $25 \%$ seu valor energético. Concluiu-se que a fibra da casca de laranja utilizada como substituto de gordura é uma alternativa viável na elaboração de sorvete light, pois manteve a maioria de suas características químicas, obtendo boa aceitabilidade sensorial.

Crizel, Araujo, Rios, Rech e Flôres (2014), identificação A19, elaboraram sorvete de limão utilizando a fibra da laranja como substituto da gordura. Foram desenvolvidas quatro tipos de fibra: F1 (casca, bagaço e semente), F2 (casca), F3 (F1 pré-tratada com hidro destilação) e F4 (F2 pré-tratada com hidro destilação). Nove formulações de sorvete foram desenvolvidas: controle (5\% de gordura - IC) e oito variando as concentrações das fibras e dos demais ingredientes (1\% - ICA, ICC, ICE, ICG; e 1,5\% ICB, ICD, ICF e ICH) de F1, F2, F3 e F4, respectivamente. Em seguida, avaliou-se a composição física, química e a aceitação sensorial. O teor de gordura da formulação controle foi 8,24\%, já nas formulações com as fibras os teores variaram de $3,84 \%$ a $4,16 \%$, redução média de $51 \%$. O teor de fibra alimentar das formulações com $1,5 \%$ de fibra de resíduos da laranja foi maior do que nas formulações com 1,0\%. A incorporação das fibras no sorvete não alterou suas propriedades principais como: parâmetros de cor, textura (dureza, adesividade, elasticidade e coesão), taxa de fusão e aceitação sensorial, em relação ao padrão. Concluiu-se que a fibra da laranja possui excelente viabilidade na substituição da gordura em formulações de sorvetes. Sua incorporação proporcionou a elaboração de um alimento com teor reduzido de gordura (light) e com presença de fibras. Esses benefícios reforçam o potencial para seu desenvolvimento, comercialização e consumo, sem interferir no nicho de mercado existente, aumentando a oferta de produtos mais saudáveis.

\subsection{Elaboração de outros subprodutos}

O estudo de Sicari, Pellicanò, Laganà e Poiana (2018), identificado como A15, utilizou farinha de casca de laranja Valência em geleia de laranja em substituição à pectina. Foram preparadas duas formulações: (P) uso de pectina tradicional com baixo teor de metoxilação, e (B) $0,3 \%$ da farinha. Avaliou-se a estabilidade, atividade antioxidante e inibição da formação de hidroximetilfurfural (HMF) em diferentes temperaturas de armazenamento $\left(20{ }^{\circ} \mathrm{C}\right.$ e $\left.35^{\circ} \mathrm{C}\right)$ durante 150 dias. Os resultados mostraram que houve um retardo na formação de HMF na formulação B, e que apresentaram teores mais elevados de fenóis totais em relação à formulação $\mathrm{P}$, variando de 110,09 a 157,32 mg/100 g. Os dados mostraram também que a formulação B acondicionada a $20{ }^{\circ} \mathrm{C}$ obteve a maior capacidade antioxidante, contudo, o armazenamento tende a reduzir essa atividade nos alimentos, fator observado em todas as formulações do estudo. As formulações acondicionadas a $20{ }^{\circ} \mathrm{C}$ mantiveram os parâmetros analíticos melhores do que as armazenadas a $35^{\circ} \mathrm{C}$. Concluiu-se que a adição de farinha da casca de laranja é uma alternativa viável à substituição da pectina na produção de geleia, não só como agente gelificante, mas também por apresentar potencial para a formulação de nutracêuticos e de conservadores alimentícios.

Silva et al. (2020), identificação A16, avaliaram a influência da substituição da gordura suína por farinha do albedo da laranja na elaboração de hambúrgueres bovinos. Foram elaboradas cinco formulações: FC, F1, F2, F3 e F4 com 0\%, 25\%, $50 \%, 75 \%$ e $100 \%$ da farinha do albedo, respectivamente. Em seguida, avaliaram a composição centesimal (farinha e hambúrgueres), físico-química e sensorial do produto final. Destaque para o elevado teor de fibra bruta na farinha do albedo $(65,87 \%)$. Os teores de proteínas e fibras aumentaram à medida que se adicionava mais farinha ao hambúrguer, inversamente proporcional ao $\mathrm{pH}$, que reduziu significativamente. As amostras F2, F3 e F4 apresentaram 3,29\%, 4,94\% e 6,59\% de fibra, respectivamente, sendo consideradas como "fonte de fibras". O teor de gordura de F2, F3 e F4 foi reduzido em mais de $25 \%$ em comparação a FC. Apenas a formulação F2 não diferiu da FC quanto ao rendimento e encolhimento no cozimento e na capacidade de retenção de água. As amostras F1 e F2 obtiveram as maiores médias para todos os atributos sensoriais analisados (cor, aparência, sabor, textura e gosto geral) e maior intenção de compra. Já F3 e F4 apresentaram textura seca e sabor frutado não característico do produto, devido à maior adição da farinha do albedo. Concluiu-se que o uso da farinha do 
albedo da laranja foi satisfatório, pois reduziu o teor lipídico e melhorou o teor de fibra dos hambúrgueres. Contudo, mais estudos são necessários para minimizar a redução do $\mathrm{pH}$ e avaliar a oxidação dos produtos, visando retardar esse processo.

Pereira et al. (2020), identificado como A22, processaram cerveja artesanal de trigo incorporando pedúnculo de caju (PC) e casca de laranja (CL), com maltes importados. Seis formulações foram elaboradas: F1 (controle), F2 (5\% PC; 0\% CL), F3 (10\% PC; 0\% CL), F4 (0\% PC; 0,6\% CL), F5 (5\% PC; 0,6\% CL) e F6 (10\% PC; 0,6\% CL). Posteriormente, realizaram as análises da composição físico-química, compostos fenólicos totais, atividade antioxidante, avaliação microbiológica e sensorial. Os resultados obtidos na caracterização físico-química e na avaliação microbiológica estavam em conformidade com a legislação. A combinação dos compostos bioativos do pedúnculo de caju e da casca de laranja contribuíram sinergicamente para a elevação da atividade antioxidante em comparação com a formulação controle. A aceitação sensorial indicou que a amostra F6, com concentrações maiores do pedúnculo e da casca apresentou maior pontuação para o atributo sabor $(7,4) \mathrm{em}$ comparação a formulação controle $(6,47)$. Os autores consideram a cerveja uma alternativa viável para o reaproveitamento desses resíduos que na maioria das vezes são descartados em locais inadequados, causando sérios problemas ambientais, demonstrando também o potencial de inovação no setor de bebidas.

\section{Conclusão}

Considera-se que a incorporação de resíduos da laranja é uma alternativa viável para elaboração de produtos alimentícios, garantindo a valorização da cultura e a redução do desperdício, que provoca prejuízos à saúde humana e ambiental. Além disso, seu reaproveitamento como forma de ingrediente, pode conferir, na maioria dos casos, melhoria das características físico-químicas, capacidade antioxidante, compostos bioativos e dos atributos sensoriais do alimento elaborado.

Apenas quatro estudos avaliaram a atividade antioxidante e/ou presença de compostos bioativos, somente um analisou os micronutrientes e nenhum trabalho elaborou rotulagem nutricional, e a mensuração do custo final unitário dos produtos. Levando em consideração que os subprodutos desenvolvidos apresentam viabilidade de comercialização, destaca-se a necessidade de avaliar essas características, possibilitando que as informações atraiam o interesse da indústria e os alimentos possam então chegar à mesa do consumidor.

Como sugestão de estudos futuros, considera-se as outras propriedades atribuídas aos resíduos da laranja e subprodutos, como capacidade antioxidante, antimicrobiana, anti-inflamatória, dentre outras, no desenvolvimento de alimentos funcionais e nutracêuticos, avaliando o custo-benefício e a aceitação sensorial do produto elaborado.

\section{Agradecimentos}

Ao Instituto Federal de Educação, Ciência e Tecnologia do Ceará (IFCE), a Fundação Cearense de Apoio ao Desenvolvimento Científico e Tecnológico (FUNCAP), ao Conselho Nacional de Desenvolvimento Científico e Tecnológico (CNPq) e a Coordenação de Aperfeiçoamento de Pessoal de Nível Superior (CAPES) pela concessão de bolsas de estudo.

\section{Referências}

Araújo, A. C. da C., \& Budoia, S. A. G. (2019). Características físico-químicas e benefícios da farinha de laranja. Nutrição Brasil, 18(1), 49-54. https://doi.org/10.33233/nb.v18i1.1625

Ayo, J. A., Ayo, V. A., \& Igweaka, C. C. (2018). Phytochemical, physicochemical and sensory quality of acha-orange peel flour blend biscuits. Publication of Nasarawa State University, 14(1), 81-90.

Benelli, P. (2010). Agregação de valor ao bagaço de laranja (Citrus sinensis L. Osbeck) mediante obtenção de extratos bioativos através de diferentes técnicas de extração. Dissertação de mestrado, Programa de Pós-graduação em Engenharia de Alimentos, Universidade Federal de Santa Catarina. Florianópolis, Brasil. 
Biolchini, J. C. de A., Mian, P. G., Natali, A. C. C., Conte, T. U., \& Travassos, G. H. (2007). Scientific research ontology to support systematic review in software engineering. Advanced Engineering Informatics, 21(2), 133-151.

Boff, C. C. e, Crizel, T. de M., Araujo, R. R. de, Rios, A. de O., \& Flôres, S. H. (2013). Desenvolvimento de sorvete de chocolate utilizando fibra de casca de laranja como substituto de gordura. Ciência Rural, 43(10), 1892-1897.

Crizel, T. de M., Araujo, R. R. de, Rios, A. de O., Rech, R., \& Flôres, S. H. (2014). Orange fiber as a novel fat replacer in lemon ice cream. Food Science and Technology, 34(2), 332-340. http://dx.doi.org/10.1590/fst.2014.0057

Crizel, T. de M., Rios, A de O., Thys, R. C. S., \& Flôres, S. H. (2015). Effects of orange by-product fiber incorporation on the functional and technological properties of pasta. Food Science and Technology, 35(3), 546-551. https://doi.org/10.1590/1678-457X.6719

FAO, FIDA, OMS, PMA \& UNICEF (2019). El estado de la seguridad alimentaria y la nutrición en el mundo 2019. Protegerse frente a la desaceleración y el debilitamiento de la economía. Roma, FAO. 256 p.

FAO. (2020). Food and Agriculture of the United Nations. Statistical Databases. http://www.fao.org/faostat/en/\#rankings/commodities_by_country

Nogueira, L. P. da S., Araújo, M. A., Melo, J. R. de L., Albuquerque, T. da N., Diniz, A. A. R., \& Lopes, M. F. (2018). Uso da farinha da casca de laranja (Citrus sinensis L.) na elaboração de pães de méis. Revista Brasileira de Gestão Ambiental, 12(1), 01-05.

Oliveira, N. A. de S., Winkelmann, D. O. V., \& Tobal, T. M. (2019). Farinhas e subprodutos da laranja sanguínea-de-mombuca: caracterização química e aplicação em sorvete. Brazilian Journal of Food Technology, 22(e2018246), 1-8. https://doi.org/10.1590/1981-6723.24618

Oliveira, V. R. de, Preto, L. T., Schmidt, H. de O., Komeroski, M., Silva, V. L. da, \& Rios, A. de O. (2016). Physicochemical and sensory evaluation of cakes made with passion fruit and orange residues. Journal of Culinary Science \& Technology, 14(2), 166-175. https://doi.org/10.1080/15428052.2015.1102787

Pereira, I. M. C., Matos Neto, J. D., Figueiredo, R. W., Carvalho, J. D. G., Figueiredo, E. A. T. de, Menezes, N. V. S. de, \& Gaban, S. V. F. (2020). Physicochemical characterization, antioxidant activity, and sensory analysis of beers brewed with cashew peduncle (Anacardium occidentale) and orange peel (Citrus sinensis). Food Science and Technology, 40(3), 749-755. https://doi.org/10.1590/fst.17319

Ramos, S. A., Pereira, R. das D., Andressa, I., Schmiele, M., \& Amaral, T. N. (2020). Desenvolvimento de cookies com coprodutos de frutas. Research, Society and Development, 9(10), e5799108918. https://doi.org/10.33448/rsd-v9i10.8918

Romero-Lopez, M. R., Osorio-Diaz, P., Bello-Perez, L. A., Tovar, J., \& Bernardino-Nicanor, A. (2011). Fiber concentrate from orange (Citrus sinensis L.) bagase: characterization and application as bakery product ingredient. International Journal of Molecular Sciences, 12(4), 2174-2186. https://doi.org/10.3390/ijms12042174

Rosolen, M. D., Bresciani, L., Sprandel, C. L., Spader, M., Klein, A. L., \& Wollmuth, J. O. M. (2018). Biscoitos tipo cookies desenvolvidos a partir de farinha de casca de laranja. Revista Destaques Acadêmicos,10(4), 8-17. http://dx.doi.org/10.22410/issn.2176-3070.v10i4a2018.1711

Santos, F. S., Lima, A. R. C., Silva, C. M., Leite, D. D. F., \& Queiroz, A. J. M. (2018). Aproveitamento da casca residual de laranja na produção de doce artesanal. In Cirne, L. E. M. R, Francisco, P. R. M., \& Farias, S. A. R. (Orgs.) Gestão Integrada de Resíduos: Universidade \& Comunidade (v.3; pp. 44-48). Campina Grande: EPGRAF. (Trabalho original publicado em 2018).

Santos, A. A. O., Santos, A. J. A. O., Silva, I. C. V. e, Leite, M. L. da C., Soares, S. da M., \& Marcellini, P. S. (2010). Desenvolvimento de biscoitos de chocolate a partir da incorporação de fécula de mandioca e albedo de laranja. Alimentos e Nutrição, 21(3), 469-480.

Santos, A. A. O., Silva, I. V. C. e, Santos, J. P. A. dos, Santana, D. G., Almeida, M. L., \& Marcellini, P. S. (2011). Elaboração de biscoitos de chocolate com substituição parcial da farinha de trigo por polvilho azedo e farinha de albedo de laranja. Ciência Rural, 41(3), 531-536. https://doi.org/10.1590/S010384782011000300028

Sdiri, S., Cuenca, J., Navarro, P., Salvador, A., \& Bermejo, A. (2020). New triploids late-maturing mandarins as a rich source of antioxidant compounds. European Food Research and Technology, 246(1), 225-237. https://doi.org/10.1007/s00217-019-03407-9

Sicari, V., Pellicanò, T. M., Laganà, V., \& Poiana, M. (2018). Use of orange by-products (dry peel) as an alternative gelling agent for marmalade production: Evaluation of antioxidant activity and inhibition of HMF formation during different storage temperature. Journal of Food Processing and Preservation, 42(2), e13429. https://doi.org/10.1111/jfpp.13429

Silva, A. S. (2015). Determinação de macrocomponentes na laranja (Citrus sinensis): variedades pera e lima, comercializados no município de São Luís MA. Monografia, Graduação em Química, Universidade Federal do Maranhão. São Luís, Brasil.

Silva, C. E. de F., Gama, B. M. V. da, Oliveira, L. M. T. de M., Araujo, L. T. de., Araujo, M. L. de, Oliveira Júnior, A. M. de, \& Abud, A. K. de S. (2016). Uso da laranja lima e seus resíduos no desenvolvimento de novos produtos. Brazilian Journal of Biosystems Engineering, 10(1), 69-96. http://dx.doi.org/10.18011/bioeng2016v10n1p69-96

Silva, I. C. V., Santos, A. A. O., Alves, A. R., Batista, M. C. de A., \& Marcellini, P. S. (2012). Fécula de mandioca e farinha de albedo de laranja na formulação de bolos de chocolate. Revista do Instituto Adolfo Lutz, 71(1), 111-117.

Silva, I. C. V., Santos, A. A. O., Santana, D. G., Santos, A. J. A. O., Leite, M. L. da C., Almeida, M. L., \& Marcellini, P. S. (2013). Avaliação da influência das variáveis açúcar, polvilho azedo e albedo de laranja na elaboração de bolos de chocolate. Brazilian Journal of Food Technology, 16(3), 175-183. http://dx.doi.org/10.1590/S1981-67232013005000022

Silva, L. B. F., Miranda, C. N., Santos, M. dos, Pereira, P. A. P., Cunha, L. R. da, Vieira, S. M., \& Gandra, K. M. B. (2020). Orange albedo flour as a fat replacer in beef burgers: adding value to citrus industry by-products. Research, Society and Development, 9(10), e1599108298. https://doi.org/10.33448/rsdv9i10.8298 
Research, Society and Development, v. 10, n. 6, e0910615373, 2021

(CC BY 4.0) | ISSN 2525-3409 | DOI: http://dx.doi.org/10.33448/rsd-v10i6.15373

Stoll, L., Flôres, S. H. \& Thys, R. C. S. (2015). Fibra de casca de laranja como substituto de gordura em pão de forma. Ciência Rural, 45(3), 567-573. https://doi.org/10.1590/0103-8478cr20131503

Storrer, D., Meireles, K. H., Perly, M. D., Lima, R. da S., \& Mattanna, P. (2017). Desenvolvimento de pão com adição de farinha de bagaço de laranja: análises sensoriais, físico-químicas e microbiológicas. Revista Eletrônica Biociências, Biotecnologia e Saúde, 9(18), 43-50.

Tozatti, P., Rigo, M., Bezerra, J. R. M. V., Córdova, K. R. V., \& Teixeira, A. M. (2013). Utilização de resíduo de laranja na elaboração de biscoitos tipo cracker. Revista Ciências Exatas e Naturais, 15(1), 135-150. https://doi.org/10.5935/RECEN.2013.01.08

Urrutia, M. D., Ramos, A. G., Horst, M., Sacks, T., Bedin, B., \& Bernardi, D. M. (2019). Formulação de bolos enriquecidos com farinhas produzidas com subprodutos da laranja. FAG Journal of Health, 1(2), 121-129. https://doi.org/10.35984/fjh.v1i2.101 